\title{
Cullin 5 is a novel candidate tumor suppressor in renal cell carcinoma involved in the maintenance of genome stability
}

María Ángeles Tapia-Laliena', Nina Korzeniewski ${ }^{1}$, Samuel Peña-Llopis ${ }^{2,6}$, Claudia Scholl ${ }^{3}$, Stefan Fröhling ${ }^{2}$, Markus Hohenfellner ${ }^{4}$, Anette Duensing ${ }^{5}$ and Stefan Duensing $\mathbb{1}^{1,4}$

\begin{abstract}
Clear cell renal cell carcinoma (ccRCC) is intimately associated with defects in ubiquitin-mediated protein degradation. Herein, we report that deficiency in the E3 ligase subunit cullin 5 (CUL5) promotes chromosomal instability and is an independent negative prognostic factor in CCRCC. CUL5 was initially identified in an RNA interference screen as a novel regulator of centrosome duplication control. We found that depletion of CUL5 rapidly promotes centriole overduplication and mitotic errors. Downregulation of CUL5 also caused an increase of DNA damage that was found to involve impaired DNA double-strand break repair. Using immunohistochemistry, CUL5 protein expression was found to be below detection level in the majority of RCCs. A re-analysis of the TCGA cCRCC cohort showed that a reduced CUL5 gene expression or CUL5 deletion were associated with a significantly worse overall patient survival. In conclusion, our results indicate that CUL5 functions as a novel tumor suppressor with prognostic relevance in cCRCC and is critically involved in the maintenance of genome stability.
\end{abstract}

\section{Introduction}

Kidney cancer affects over 300,000 people worldwide annually and is one of the most lethal urological malignancies once metastatic ${ }^{1}$. Clear cell renal cell carcinoma (ccRCC) is the most common histological subtype and is thought to arise from cells lining the proximal tubule of the nephron ${ }^{2}$.

Like most solid tumors, ccRCC is characterized by chromosomal instability including numerical and structural chromosomal alterations ${ }^{3}$. Some of these alterations such as the loss of chromosome $3 p$ are highly

\footnotetext{
Correspondence: Stefan Duensing (stefan.duensing@med.uni-heidelberg.de) 'Section of Molecular Urooncology, Department of Urology, University of Heidelberg School of Medicine, Im Neuenheimer Feld 517, D-69120 Heidelberg, Germany

${ }^{2}$ Department of Translational Medical Oncology, National Center for Tumor Diseases (NCT) Heidelberg, German Cancer Research Center (DKFZ), Im Neuenheimer Feld 460, D-69120 Heidelberg, Germany

Full list of author information is available at the end of the article. Co-first authors: María Ángeles Tapia-Laliena, Nina Korzeniewski

Co-senior authors: Anette Duensing, Stefan Duensing
}

characteristic for $\mathrm{ccRCC}^{4,5}$. While loss of chromosome $3 p$ has been suggested to represent an early event in $\mathrm{ccRCC}^{4}$, there is an association between chromosomal complexity and metastatic disease as highlighted by the frequent coincidence of loss of chromosomes $9 p$ and $14 q$ in advanced stage disease ${ }^{6}$. Whole chromosome copy number changes (aneuploidy) are also frequent findings in ccRCC, which, together with structural changes and single-nucleotide variants ${ }^{7}$ contribute to the extensive intratumoral genetic heterogeneity characteristic of $\operatorname{ccRCC}^{8,9}$.

In general, numerical and structural chromosomal aberrations are caused by mitotic defects and errors in DNA damage repair, respectively, which frequently coincide in cancer cells ${ }^{10}$.

In ccRCC, the inactivation of the $V H L$ tumor suppressor gene, which occurs in the large majority of patients, has been shown to lead to defective mitoses and also to interfere with DNA double-strand break (DSB) repair ${ }^{11,12}$. The pVHL protein is part of a protein complex that

\section{(c) The Author(s) 2019}

(c) (i) Open Access This article is licensed under a Creative Commons Attribution 4.0 International License, which permits use, sharing, adaptation, distribution and reproduction in any medium or format, as long as you give appropriate credit to the original author(s) and the source, provide a link to the Creative Commons license, and indicate if changes were made. The images or other third party material in this article are included in the article's Creative Commons license, unless indicated otherwise in a credit line to the material. If material is not included in the article's Creative Commons license and your intended use is not permitted by statutory regulation or exceeds the permitted use, you will need to obtain permission directly from the copyright holder. To view a copy of this license, visit http://creativecommons.org/licenses/by/4.0/. 
includes elongin $\mathrm{B}$, elongin $\mathrm{C}, \mathrm{Rbx} 1$ and cullin 2 and functions as E3 ubiquitin ligase ${ }^{13-15}$. Cullin RING E3 ubiquitin ligases (CRLs) constitute the major subfamily of E3 ligases and play an important role in the ubiquitinmediated protein turnover in cells. CRLs are characterized by a common cullin-containing scaffold protein ${ }^{15}$. There are eight human cullin subunits (CUL1, $-2,-3,-4 \mathrm{~A},-4 \mathrm{~B}$, $-5,-7$ and PARC) which orchestrate the assembly of unique ubiquitin ligase complexes. All CRLs consist of a cullin-backbone, a zinc-binding RING-domain containing protein, which recruits the ubiquitin-conjugating E2 enzyme, and an adaptor protein that binds interchangeable substrate recognition subunits, which provide target specificity to each individual CRL ${ }^{15-17}$.

Another main tumor suppressor gene in ccRCC is the deubiquitinase BAP1, which is inactivated in about $15 \%$ of patients ${ }^{18}$ and, among other functions, promotes DNA DSB repair ${ }^{19}$. Whether and to what extent the loss of additional tumor suppressors involved in ubiquitin-proteasomemediated protein degradation contribute to chromosomal instability in ccRCC is a matter of ongoing research ${ }^{20}$.

Herein, we show that CUL5 is a novel candidate tumor suppressor in ccRCC. Our results show that CUL5 is critically involved in the regulation of centriole duplication and DNA damage repair, and that loss of expression is a negative prognostic factor in ccRCC patients. Our findings highlight the central role of CRLs, including CUL5, in RCC development and progression.

\section{Results}

\section{Downregulation of CUL5 promotes centriole overduplication}

To explore the role of cullins in the maintenance of mitotic fidelity, we performed a small interfering RNA (siRNA) mini-screen of seven human cullin subunits. Protein knock-down was performed in U-2 OS cells stably expressing centrin-green fluorescent protein (U-2 OS/ centrin-GFP; Fig. 1a; Suppl. Figure 1). This allows the visualization of centrioles, the core forming units of centrosomes, which serve as the major microtubuleorganizing centers in most mammalian cells in interphase and mitosis. We found that knock-down of CUL5 leads to an overduplication of centrioles in a very high percentage of cells $(56.9 \%, p \leq 0.001$; Fig. 1a). This increase is among the highest reported so far after transient manipulation of cells and only comparable to PLK4 (pololike kinase 4) overexpression, one of the strongest stimuli for centriole overduplication known so $\mathrm{far}^{21,22}$. Following depletion of CUL5, we observed several centriole overduplication defects including centriole multiplication and daughter-daughter pairs (Fig. 1a, bottom panels).

We also used a dominant-negative truncation mutant of CUL5 (DN-CUL5), which effectively reduces E3 ligase activity $^{23}$, to transiently transfect U-2 OS/centrin-GFP cells. An increase in centriole overduplication from $6.6 \%$ in controls to $29 \%$ in DN-CUL5 transfected cells ( $p \leq$ 0.001; Fig. 1b) was detected.

In order to prove that CUL5-based E3 ubiquitin ligase activity also restrained centriole biogenesis in nontransformed cells, we generated a CUL5 short-hairpin RNA (shRNA)-expressing stable cell lines using normal B) fibroblasts expressing the catalytic subunit of telomerase (BJ/TERT) and analyzed centriole numbers. Knock-down of CUL5 by shRNA increased the number of cells with centriole overduplication to $21.5 \%$ as compared to $4 \%$ with the shRNA control vector $(p \leq 0.001$; Suppl. Figure 2). This confirms our previous results with siRNA experiments in U-2 OS centrin-GFP cells.

We next analyzed the cellular localization of CUL5 in U-2 OS/centrin-GFP cells by fluorescence microscopy. We found CUL5 to localize to centrioles (Fig. 1c). This co-localization pattern between centrin-GFP and CUL5 suggested that CUL5 may be present mostly at mature centrioles. Co-staining of CUL5 and CEP170, a marker for older, mature centrioles ${ }^{24}$, in U-2 OS/centrinGFP cells confirmed that CUL5 does show a more predominant expression at older, mature centrioles (Fig. 1d).

\section{Knock-down of CUL5 produces a genuine centriole duplication defect}

When we performed an immunofluorescence microscopic analysis of U-2 OS/centrin-GFP cells for $\gamma$-tubulin after depletion of CUL 5 by siRNA, we found a sixfold increase in the number of $\gamma$-tubulin dots per cell (not shown) underscoring that supernumerary centrioles induced by knock-down of CUL5 undergo maturation and can hence potentially function as microtubuleorganizing centers in cells.

Genuine centriole overduplication is characterized by the presence of one or two mature centrioles and an abnormal number of immature daughter centrioles ${ }^{21,24}$. In contrast, centriole accumulation is characterized by the presence of multiple maternal centrioles with a normal mother-daughter centriole ratio ${ }^{21,24}$. We found that only a small fraction of cells (10\%; Fig. 1e) in asynchronously growing, CUL5-depleted U-2 OS/centrin-GFP cells contained an abnormal number of CEP170-positive centrioles (>2 CEP170-positive centrioles) in the presence of an increased number of immature (CEP170-negative) centrioles (Fig. 1e), demonstrating that aborted mitosis or cytokinesis errors are not a major mechanism behind the centriole overduplication in cells depleted of CUL5.

Taken together, these results suggest that CUL5 depletion results in an increase in supernumerary centrioles through genuine disruption of the centriole duplication cycle and that a significant fraction of these overduplicated centrioles are capable of recruiting $\gamma$ tubulin, indicating that they are functional. 


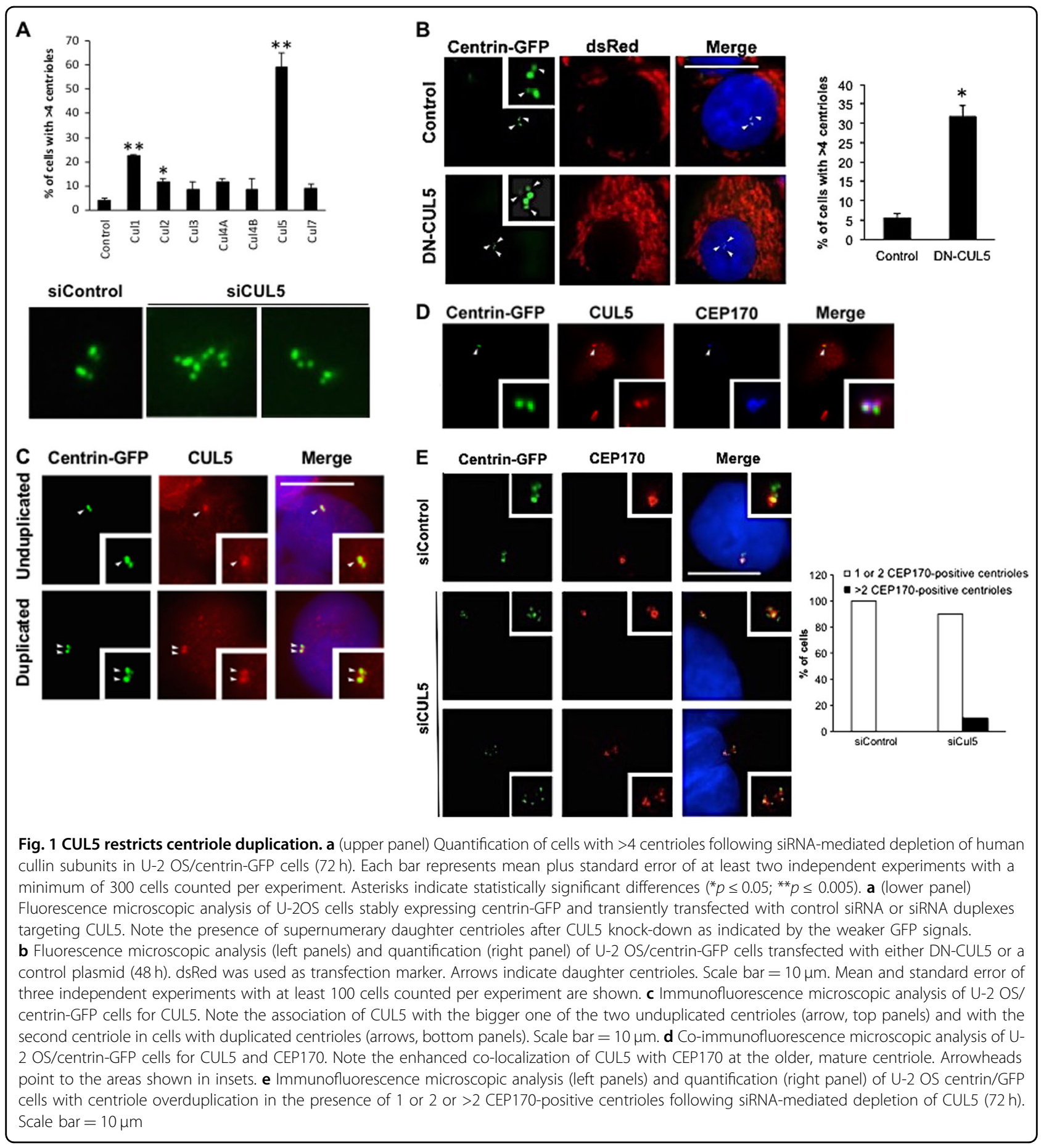

\section{CUL5 is necessary to maintain mitotic fidelity}

We next determined the consequences of CUL5 knockdown on mitotic fidelity. CUL5 depletion significantly increased the percentage of cells exhibiting abnormal mitosis (multipolar and pseudo-bipolar combined) from $19 \%$ in control cells to $38 \%$ in CUL5 siRNA-transfected cells $(p \leq 0.001)$. Whereas multipolar mitoses increased from $1 \%$ in controls to $5 \%$ in CUL5-depleted cells, the proportion of pseudo-bipolar mitoses increased from $18 \%$ in controls to $33 \%$ in CUL5-depleted cells ( $p \leq 0.05$; Fig. 2a).

These results further underscore that supernumerary centrioles induced by CUL5 depletion are functional and can promote abnormal mitoses, thereby potentially promoting chromosomal instability in daughter cells.

Flow cytometric analysis of the DNA content of CUL5depleted cells showed an increase in the number of cells 

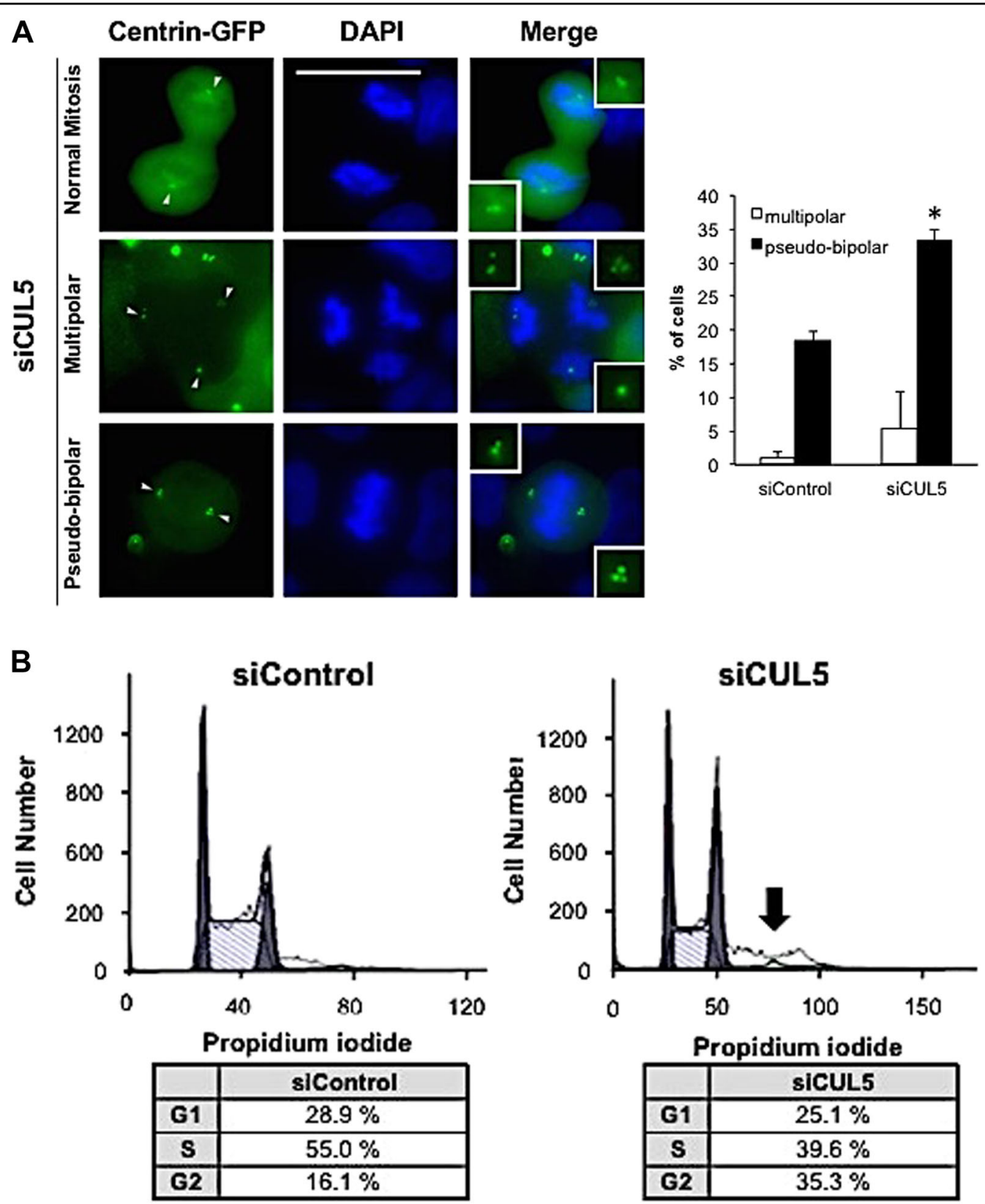

Fig. 2 CUL5 is required to maintain mitotic fidelity. a Fluorescence microscopic analysis (left panel) and quantification (right panel) of abnormal mitotic cells after siRNA-mediated depletion of CUL5 in U-2OS/centrin-GFP cells ( $96 \mathrm{~h}$ ). Insets show the spindle poles. Scale bar $=10 \mu \mathrm{m}$. Each bar represents mean plus standard error of at least two independent experiments with a minimum of 100 cells counted per experiment. $\mathbf{b}$ Flow cytometric analysis of U-2 OS/centrin-GFP cells following siRNA-mediated depletion of CUL5 (siCUL5) or control siRNA (siControl) for $72 \mathrm{~h}$. Arrow indicates poly-/aneuploid cells. Asterisks indicate statistically significant differences $\left({ }^{*} p \leq 0.05\right)$

in the $\mathrm{G}_{2} / \mathrm{M}$ phase of the cell division cycle $(35.3 \%)$ compared to control siRNA-treated cells $(16.1 \%)(p \leq$ 0.001; Fig. 2b). There was also an increase of cells with $>4 \mathrm{~N}$ DNA content, indicating the presence of polyploid/ aneuploid tumor cells following CUL5 depletion.

Moreover, we detected signs of structural DNA aberrations in cells with abrogated CUL5 function, including lagging chromosomes, anaphase bridges and micronuclei
(Fig. 3a). In particular, there was a significant increase of cells containing micronuclei from $3.3 \%$ in controls to $12.2 \%$ in cells transfected with DN-CUL5 $(p \leq 0.0001$; Fig. 3a).

Taken together, our results show that depletion of CUL5 rapidly causes mitotic defects and chromosome segregation errors but also alterations suggestive of additional structural chromosomal damage. 


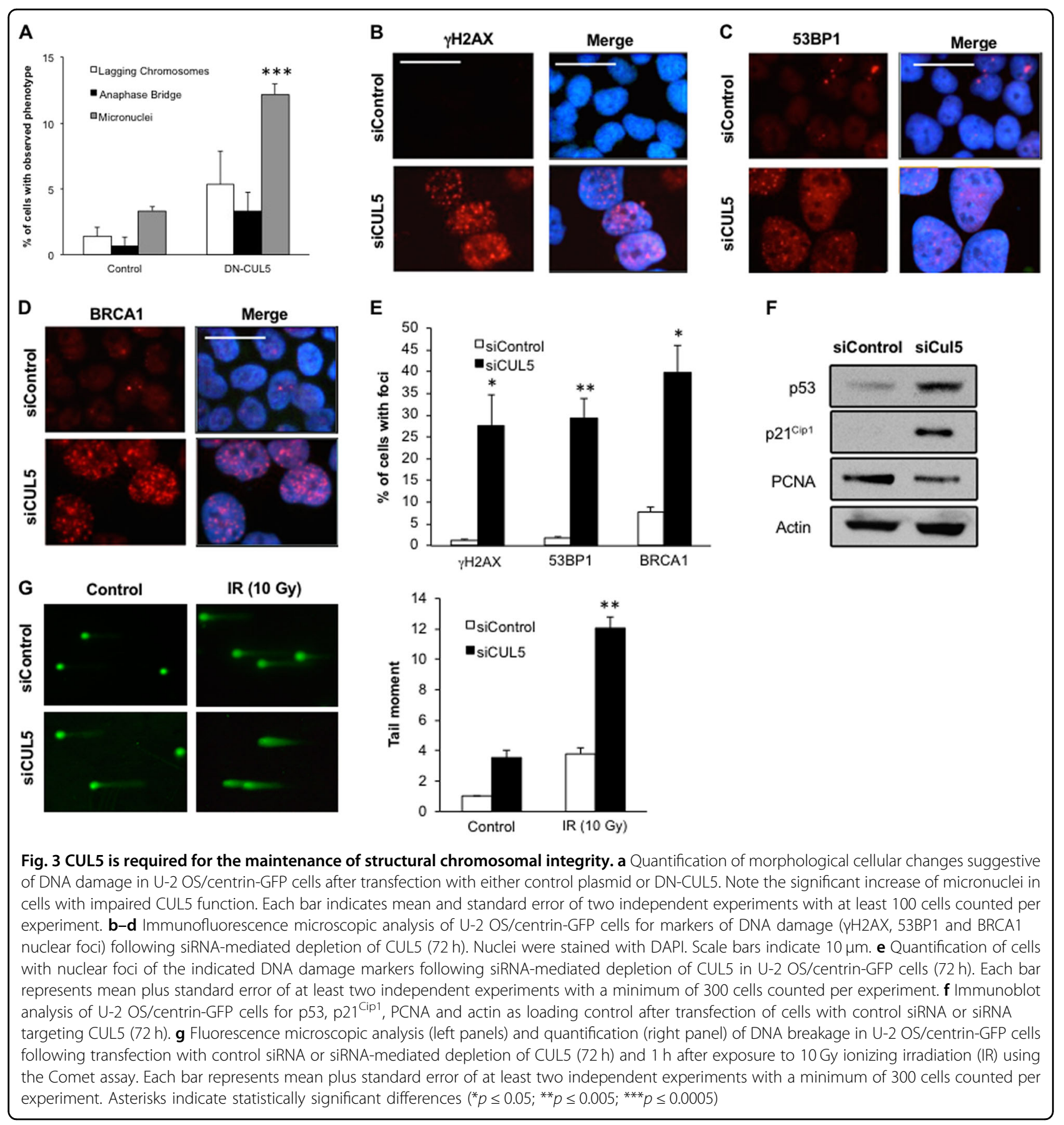

\section{CUL5 is required for DNA damage repair}

Having shown that CUL5 depletion leads to a significant increase of cells with micronuclei, we next explored a possible role of CUL5 in the cellular response to DNA damage.

First, we examined the formation of DNA damageassociated foci containing $\gamma \mathrm{H} 2 \mathrm{AX}$ (Fig. 3b), 53BP1 (Fig. 3c) or BRCA1 (Fig. 3d) after depletion of CUL5 by siRNA in U-2 OS/centrin-GFP cells. We detected a 22.9fold increase of $\gamma \mathrm{H} 2 \mathrm{AX}$ foci (from $1.2 \%$ to $27.5 \%$; Fig. 3e), a 16.2 -fold increase of $53 \mathrm{BP} 1$ foci (from $1.8 \%$ to $29.2 \%$; Fig. 3e), and a 5.1-fold increase of BRCA1 foci (from $7.9 \%$ to $39.9 \%$; Fig. 3e). This DNA damage response was accompanied by a cellular stress response including an upregulation of p53 and its transcriptional target p21 ${ }^{\text {Cip } 1}$ as well as reduced cellular proliferation (Fig. $3 \mathrm{f}$ ).

We next asked whether CUL5 depletion can directly induce DNA strand breaks or whether it rather interferes with the repair of DNA strand breaks. To this end, we performed a Comet assay to quantify the DNA damage in 
U-2 OS cells after CUL5 siRNA transfection and exposure to $10 \mathrm{~Gy}$ of ionizing radiation (IR; Fig. 3g). In particular, the Comet tail moment, which is defined by the product of the tail length and the fraction of total DNA in the tail, represents a suitable measure of the extent of DNA damage. We found an increase of the tail moment in CUL5 siRNA-transfected cells compared to controls in the absence of IR, suggesting that CUL5 depletion by itself can induce DNA breakage. However, a significant increase of the tail moment was detected after prior exposure to IR, suggesting that CUL5 may also interfere with the DNA strand break repair after exogenous DNA damage (Fig. $3 g)$.

Taken together, these results show that CUL5 downregulation promotes DNA breakage and interferes with DNA break repair.

\section{Frequent loss of CUL5 protein expression in renal cell carcinoma}

Since CUL5 shares the substrate recognition adaptor and the SOCS/BC box protein substrate receptor with CUL2, which has already been implied in renal carcinogenesis through von Hippel-Lindau (VHL) ${ }^{25,26}$, we decided to study the potential role of CUL5 in RCC progression in greater detail.

First we analyzed the protein expression of CUL5 in RCC tissue samples by immunohistochemistry (Fig. 4a). Whereas normal kidney tissue was consistently positive for CUL5, only 4 of 71 ccRCCs, 1 of 5 chromophobe RCCs and no papillary RCC showed expression of CUL5 (Fig. 4a, right panel).

We next suppressed CUL5 messenger RNA (mRNA) level in normal human renal epithelial cells (hRECs) using siRNA and found that depletion of CUL5 promoted centriole overduplication in $15.6 \%$ of cells compared to $6.6 \%$ of control cells $(p \leq 0.0001$; Fig. $4 \mathrm{~b})$. Next, we examined CUL5 mRNA level in normal human kidney cells versus the metastatic renal carcinoma cell line ACHN, which shows mixed papillary and clear cell features ${ }^{27}$. ACHN cells have also been tested for mutations in CUL5 and were found to be negative except for a silent mutation in exon $3^{28}$. ACHN cells showed a significantly reduced CUL5 mRNA expression than normal human kidney cells cells $(p \leq 0.005$; Fig. $4 c)$. In line with a reduced amount of CUL5 mRNA, the ACHN cell line also exhibited a high percentage of cells, i.e., $21.5 \%$ with centriole overduplication compared to $6.5 \%$ in normal human kidney cells ( $p \leq 0.05$; Fig. $4 \mathrm{~d})$.

Since the metastatic ACHN cells present low CUL5 mRNA levels together with a high percentage of centriole overduplication, compared to normal hRECs, CUL5 may be an important factor for the progression of renal carcinoma.

\section{Loss of CUL5 gene expression is associated with an unfavorable prognosis in RCC patients}

To further corroborate the notion that a loss of CUL5 may play a role in the progression of ccRCC, we analyzed The Cancer Genome Atlas (TCGA) Kidney Renal Clear Cell Carcinoma (KIRC) cohort, a large patient cohort $(n>$ 400) for which both clinical and genomic data are available (Fig. 5).

Patients harboring tumors with low expression levels of CUL5 displayed a shorter survival (a median of 5.2 years) compared to high CUL5 expressing tumors (a median of 7.5 years) ( $p=0.003$, Fig. 5a). Patients with chromosomal deletions in the CUL5 locus showed a significantly impaired cancer-specific survival (median of 2.7 years compared to 6.5 years, $p=0.0012$, Fig. $5 \mathrm{~b}$ ).

To investigate the potential of CUL5 levels as biomarker of prognosis, we performed univariate Cox regression models on the clinical variables and CUL5 gene expression levels (Table 1). Variables that were significant in the univariate Cox model were entered into an unsupervised stepwise forward conditional multivariate Cox analysis to identify independent predictors of survival. Advanced age (higher than median), high grade, metastasis at diagnosis $(\mathrm{M}+)$, high tumor stage and low CUL5 expression were independently associated with poor survival in ccRCC patients by being present in the final step of the multivariate Cox model. The same variables were retained in a stepwise backward conditional multivariate analysis.

In summary, low CUL5 gene expression is an independent negative prognostic factor in ccRCC.

\section{Discussion}

CUL5 was originally cloned as vasopressin-activated calcium-mobilizing (VACM-1)-encoding gene ${ }^{29,30}$. CUL5 is the least conserved of the cullin family members ${ }^{31,32}$ and it has gained attention through its role in the CRLmediated degradation of APOBEC3G by human immunodeficiency virus Vif to thwart host cell antiviral defense mechanisms ${ }^{33}$. In cancer, downregulation of CUL5 has been found in a number of entities including breast can$\operatorname{cer}^{30,34,35}$, endometrial cancer ${ }^{36}$, cervical cancer ${ }^{37}$ and Bcell chronic lymphocytic leukemia ${ }^{35}$.

CUL5 is expressed in normal renal collecting tubule cells $^{38}$ and the genomic locus of the CUL5 gene, chromosome 11q22-23, has recently been implicated as a risk locus for RCC in a genome-wide association study ${ }^{39}$.

In the present report, we show that a reduced CUL5 gene expression or CUL5 deletion is associated with significantly impaired overall survival in ccRCC patients and with more rapid tumor progression, respectively. Remarkably, low CUL5 expression was an independent prognostic factor in ccRCC, a tumor entity for which patient risk stratifiers are urgently needed. 

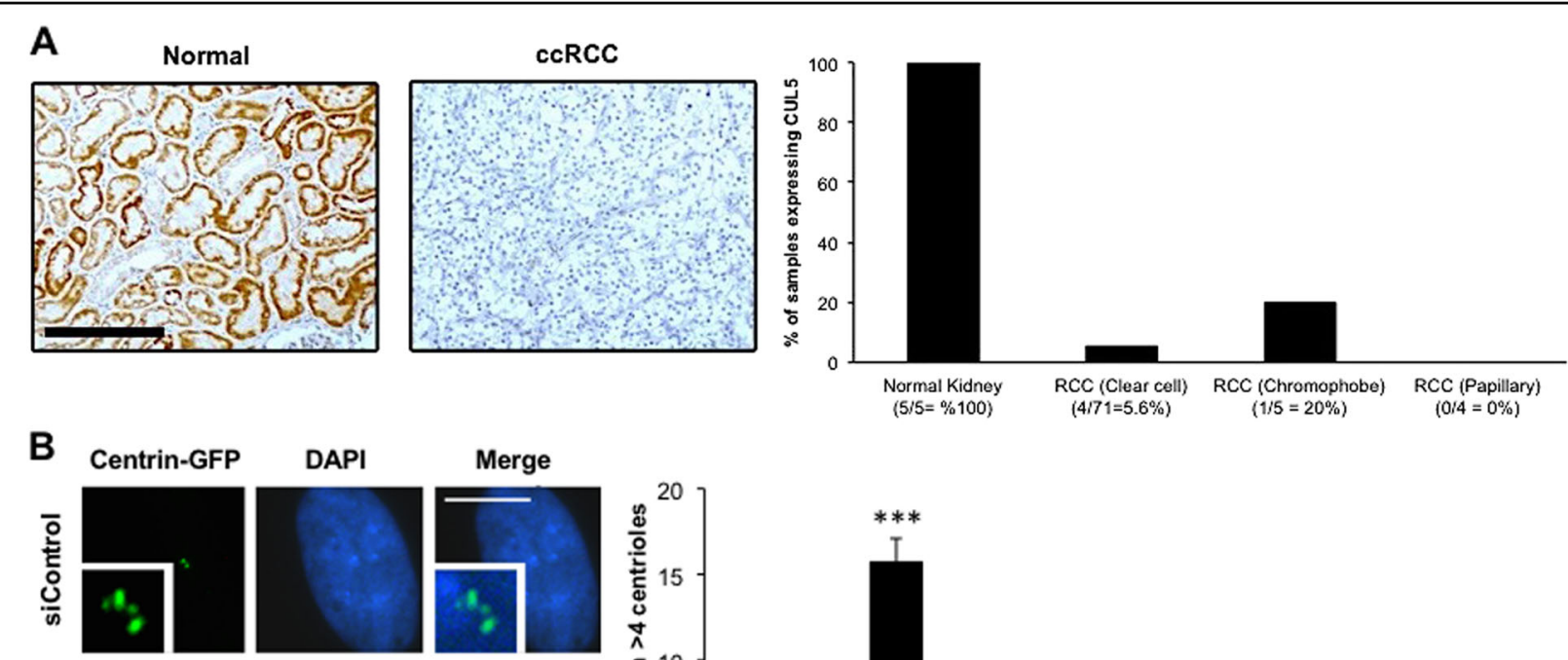

Merge
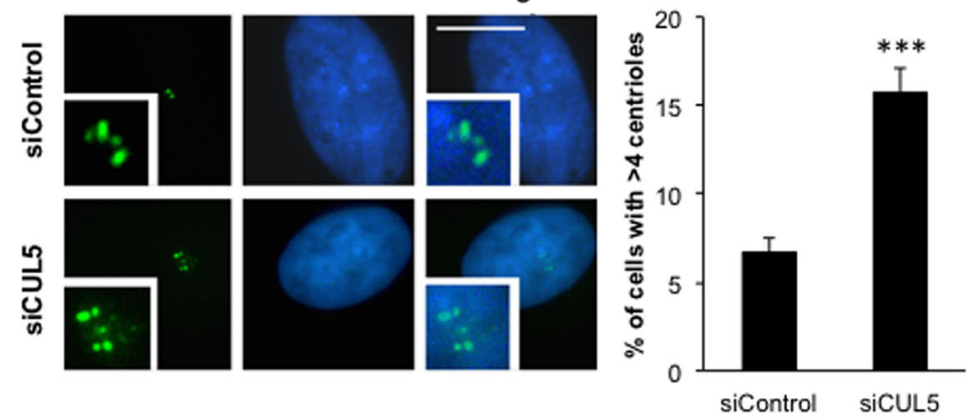

C

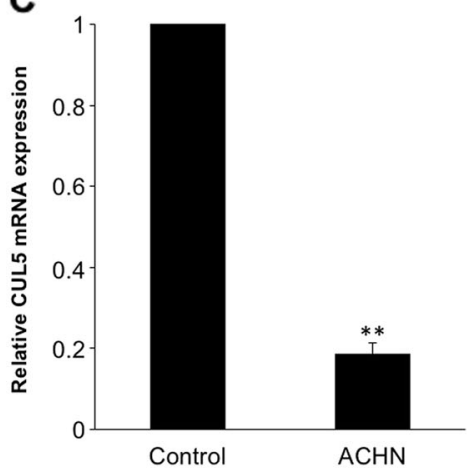

D

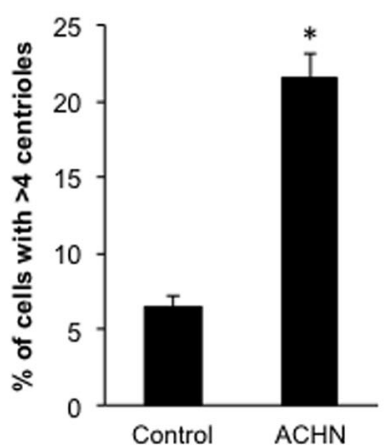

Fig. 4 Loss of CUL5 expression in renal cancer. a Immunohistochemical staining (left panels) and quantification (right panel) of CUL5 expression using a renal cell carcinoma tissue microarray. Representative immunostainings of CUL5 in human normal kidney and a cCRCC are shown. Scale bar $=250 \mu \mathrm{m}$. b Fluorescence microscopic analysis (left panels) and quantification (right panel) of normal human renal epithelial cell (hRECs) for centriole numbers following siRNA-mediated depletion of CUL5 and co-transfection with a plasmid that expresses centrin-GFP (72 h). Each bar represents mean plus standard error of at least two independent experiments with a minimum of 300 cells counted per experiment. c Quantitative real-time reverse transcriptase polymerase chain reaction (qRT-PCR) for CUL5 mRNA expression in normal human kidney cells (HEK293) used as controls and ACHN cells. $\beta$-Actin was used as a control housekeeping gene. Five replicates were performed. $\mathbf{d}$ Quantification of cells with spontaneous centriole overduplication in primary human renal epithelial cells (Control) in comparison to ACHN cells. Centrioles were visualized by transfection with a plasmid encoding centrin-GFP (48 h). Mean and standard error of two independent experiments with at least 100 cells counted per experiment are shown. Asterisks indicate statistically significant differences $\left({ }^{*} p \leq 0.05 ;{ }^{* *} p \leq 0.005 ;{ }^{* * *} p \leq 0.0005\right)$

Mechanistically, we show that loss of CUL5 can rapidly disrupt mitotic fidelity and induce structural chromosomal damage, very likely with an attenuation of DNA DSB repair as a strong contributing factor. In this regard, CUL5 loss may contribute to the extensive intratumoral heterogeneity that characterizes ccRCC and that is driven by genomic instability ${ }^{8,9,40}$. Chromosome 11 q loss has not been found to represent a hotspot for somatic copy number alterations in the TRACERx cohort $^{3}$ and our findings showing a CUL5 deletion in approximately $10 \%$ of ccRCC patients are in line with this finding. Nevertheless, if such a CUL5 loss is present, it confers a more rapid progression towards a lethal disease outcome (Fig. $4 b)$.

CUL5 has previously been implicated to function as a tumor suppressor by regulating cellular proliferation ${ }^{38}$. 

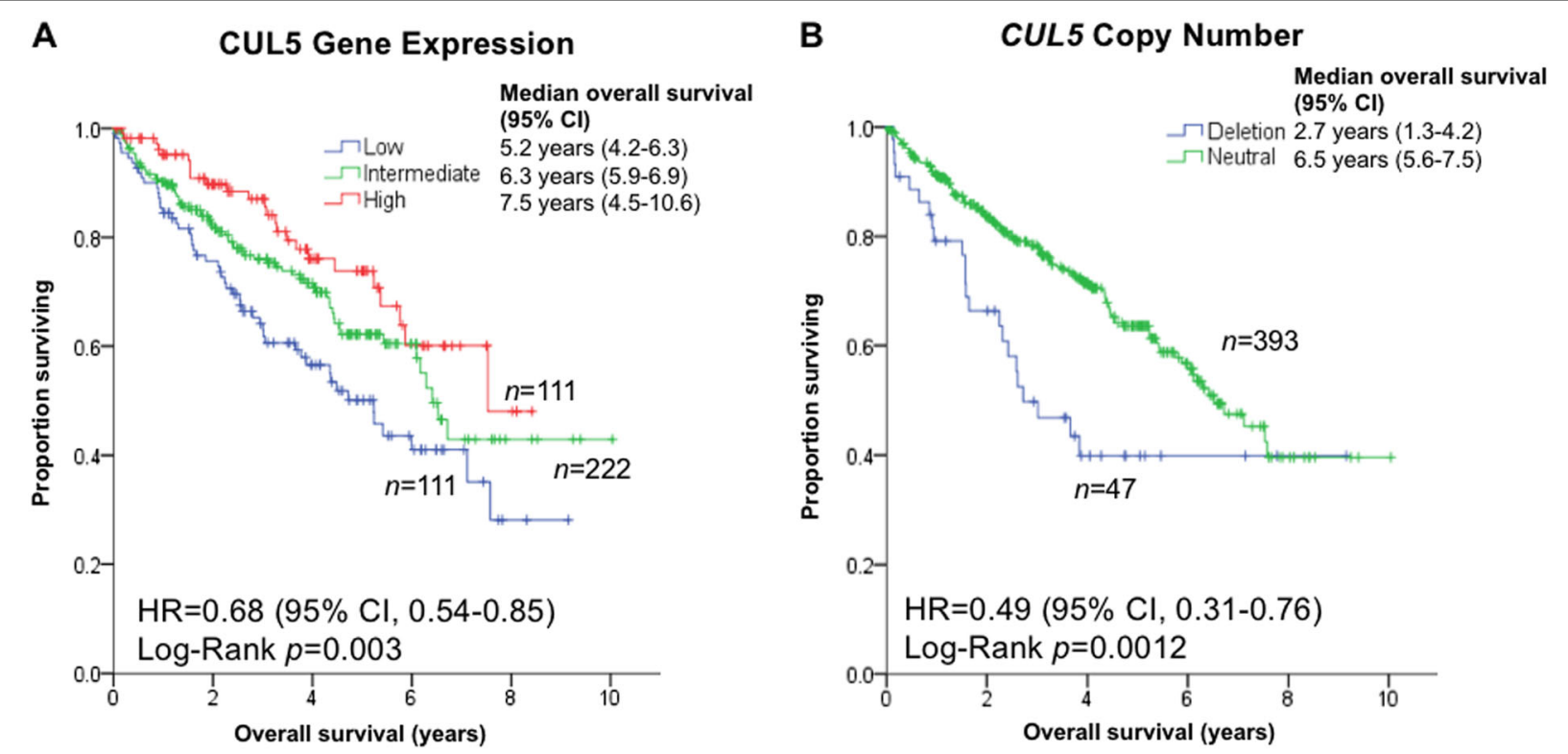

Fig. 5 Low CUL5 expression is associated with impaired survival in patients with ccRCC. a Kaplan-Meier survival curve of KIRC-TCGA patients showing low CUL5 gene expression (1st quartile, blue), which is associated with significantly worse overall overall survival when compared to patients with high CUL5 expression levels (4th quartile, red). Second and third quartiles are shown combined in green. $\mathbf{b}$ Kaplan-Meier survival curve of KIRC-TCGA patients with a CUL5 chromosomal deletion in comparison to patients with no loss of the CUL5 gene. Note the significantly worse overall survival in patients with a CUL5 deletion

Table 1 Univariate and multivariate Cox regressions of the clinical variables and CUL5 expression from the KIRC-TCGA dataset

\begin{tabular}{|c|c|c|c|c|c|c|}
\hline & Uni & & & $\begin{array}{l}\text { Multivariate } \\
\text { analysis }\end{array}$ & & \\
\hline Variable & HR & $95 \% \mathrm{Cl}$ & $P$ & HR & $95 \% \mathrm{Cl}$ & $P$ \\
\hline Age (>median) & 2.0 & $1.4-2.7$ & $8 \cdot 10^{-5}$ & 2.0 & $1.3-3.3$ & 0.004 \\
\hline Gender (male) & 0.8 & $0.6-1.2$ & 0.32 & & & \\
\hline High grade (3-4 vs. $1-2)$ & 2.3 & $1.6-3.3$ & $7 \cdot 10^{-6}$ & 1.7 & $1.0-2.9$ & 0.03 \\
\hline High tumor size (>median) & 2.7 & $1.9-3.8$ & $4 \cdot 10^{-8}$ & & & \\
\hline High T (3-4 vs. 1-2) & 3.1 & $2.2-4.3$ & $2 \cdot 10^{-11}$ & & & \\
\hline $\mathrm{N}+$ & 3.4 & $1.7-7.0$ & $6 \cdot 10^{-4}$ & & & \\
\hline$M+$ & 4.4 & $3.2-6.2$ & $3 \cdot 10^{-18}$ & 3.1 & $1.8-5.4$ & $5 \cdot 10^{-5}$ \\
\hline High stage (III-IV vs. I-II) & 3.9 & $2.8-5.6$ & $10^{-14}$ & 2.1 & $1.2-3.8$ & 0.009 \\
\hline Low CUL5 expression & 1.7 & $1.2-2.4$ & 0.003 & 1.7 & $1.0-2.7$ & 0.03 \\
\hline CUL5 chromosomal deletion & 2.0 & $1.3-3.2$ & 0.002 & & & \\
\hline
\end{tabular}

$H R$ hazard ratio, $\mathrm{Cl}$ confidence interval

CUL5 has been shown to be expressed in nonproliferating endothelial cells and downregulated during angiogenesis $^{41}$. It is hence possible that downregulation or loss of CUL5 in ccRCC may further fuel neo-angiogenesis, which plays a central role in ccRCC driven by VHL loss, thus promoting tumor progression.
CUL5 has been shown to restrict Src activity ${ }^{42}$. Src is a potent inducer of tumorigenesis and has been implicated in both the regulation of cell division and DNA damage repair $^{43,44}$. Whether Src or a different substrate of CUL5based E3 ligase activity in fact mediates the observed 
cellular effects of a CUL5 loss on genome stability is the subject of future experiments.

Taken together, our results demonstrate that CUL5 is a novel candidate tumor suppressor in ccRCC that is involved in the maintenance of genome stability and has independent prognostic value in ccRCC patients.

\section{Materials and methods}

\section{Cell culture and transfection}

Human U-2 OS and HEK293 cells were obtained from ATCC and maintained in Dulbecco's modified Eagle's medium (Cambrex, Walkersville, MD) supplemented with $10 \%$ fetal bovine serum (PAA, Pasching, Germany), $50 \mathrm{units} / \mathrm{ml}$ penicillin and $50 \mathrm{mg} / \mathrm{ml}$ streptomycin (PAA, Pasching, Germany). BJ/TERT fibroblasts were kindly provided by Ole Gjoerup (Dana-Farber Cancer Institute, Boston, MA, USA) and were maintained as reported previously $^{21}$. U-2 OS and BJ/TERT cells were engineered to stably express a centrin-GFP-encoding construct (kindly provided by Michel Bornens, Institut Curie, Paris, France $\left.{ }^{45}\right)$. ACHN cells were obtained from Cell Line Services (Eppelheim, Germany) and maintained in Eagle's minimal essential medium supplemented with $10 \%$ fetal bovine serum (PAA, Pasching, Germany), 50 units $/ \mathrm{ml}$ penicillin and $50 \mathrm{mg} / \mathrm{ml}$ streptomycin (PAA, Pasching, Germany). Normal human renal epithelial cells were obtained from Lonza (Basel, Switzerland) and maintained in Clonetics ${ }^{\mathrm{Tu}}$ REGM $^{\mathrm{su}}$ Renal Epithelial Cell Growth Medium (Lonza, Basel, Switzerland). For transient transfections of U-2 OS (48 h), DN-CUL5 (provided by Wade Harper through Addgene) or empty vector controls were used and transfected by lipofection (Fugene 6; Roche). A vector encoding red fluorescent protein targeted to mitochondria (dsRed; BD Biosciences Clontech, Palo Alto, CA, USA) was used as transfection control. For transient transfection of hRECs $(48 \mathrm{~h})$, centrin-GFP was used and transfected using the Neon ${ }^{\circledR}$ Transfection System for Electroporation according to the manufacturer's protocol (Invitrogen, Carlsbad, CA, USA).

\section{Immunofluorescence microscopy}

Cells grown on $10 \mathrm{~mm}$ coverslips were permeabilized with $1 \%$ Triton-X-100 for $15 \mathrm{~min}$, washed in phosphatebuffered saline (PBS) and then fixed in $4 \%$ paraformaldehyde/PBS followed by blocking in 10\% normal donkey serum (Jackson Immunoresearch, West Grove, PA, USA). Cells were incubated with primary antibody overnight followed by incubation with a Rhodamine Redor Coumarin (AMCA)-conjugated secondary antibody (Jackson Immunoresearch, UK) for $2 \mathrm{~h}$ and mounted with 4',6-diamidino-2-phenylindole (DAPI). Cells were analyzed using an Olympus AX70 epifluorescence microscope equipped with a SpotRT digital camera. Antibodies used were mouse anti-BRCA1, rabbit anti-CUL5 and rabbit anti-53BP1 obtained from Santa Cruz Biotechnology (Santa Cruz, CA, USA). An anti- $\gamma \mathrm{H} 2 \mathrm{AX}$ antibody was obtained from Millipore. Mouse anti-Cep170 was a kind gift from Erich A. Nigg (Biozentrum, University of Basel, Switzerland) ${ }^{24}$.

\section{siRNA and shRNA}

Synthetic RNA duplexes to reduce CUL5 protein expression were used (Flexitube, Qiagen, Valencia, CA, USA; Hs-CUL5_1 sense strand 5'-GGUUUGAAUCAGUCACCUATT-3', antisense strand 5'-UAGGUGACUGAUUCAAACCTG-3') according to the manufacturer's protocol. For the cullin siRNA mini-screen, siRNAs were obtained from Qiagen (Flexitube; target sequences available upon request). shRNA vectors for CUL5 (TR313638) were obtained from OriGene Technologies, Inc. (Rockville, MD, USA).

\section{Immunoblot analysis}

Cell lysates were prepared using an NP-40-based lysis buffer (1\% NP-40, $50 \mathrm{mM}$ Tris- $\mathrm{HCl}, \mathrm{pH} 8.0,100 \mathrm{mM}$ sodium fluoride, $30 \mathrm{mM}$ sodium pyrophosphate, $2 \mathrm{mM}$ sodium molybdate, $5 \mathrm{mM}$ EDTA, $2 \mathrm{mM}$ sodium orthovanadate in $\left.\mathrm{dH}_{2} \mathrm{O}\right)$ containing protease inhibitors $(10 \mu \mathrm{g} /$ $\mathrm{ml}$ aprotinin, $10 \mu \mathrm{g} / \mathrm{ml}$ leupeptin, $1 \mu \mathrm{M}$ phenylmethylsulfonyl fluoride, $2 \mathrm{M}$ vanadate). After $1 \mathrm{~h}$ rotation at $4{ }^{\circ} \mathrm{C}$, lysates were cleared by centrifugation for $30 \mathrm{~min}$ at $13,000 \mathrm{rpm}$ at $4^{\circ} \mathrm{C}$. Protein concentrations were determined using the Bradford assay (Bio-Rad Laboratories, Hercules, CA, USA). Then, $30 \mu \mathrm{g}$ of protein was loaded on a $4-12 \%$ Bis-Tris or $3-8 \%$ Tris-Acetate gel (Invitrogen, Carlsbad, CA, USA) and blotted onto a nitrocellulose membrane.

Antibodies directed against CUL5, p21 ${ }^{\mathrm{Cip} 1}$ (F-5), p53 (DO-1) and PCNA (PC10) were obtained from Santa Cruz Biotechnology (Santa Cruz, CA, USA). An antibody directed against actin (AC-42) was purchased from Sigma (St. Louis, MO, USA).

\section{Quantitative real-time PCR}

For quantitative real-time polymerase chain reaction (qPCR), RNA was extracted using the RNeasy Mini Kit (Qiagen, Valencia, CA, USA) according to the manufacturer's protocol. Extracted RNA was first treated with DNase I enzyme (Fermentas, St. Leon-Rot, Germany) according to the manufacturer's protocol to remove any contaminating traces of genomic DNA. Complementary DNA (cDNA) was then transcribed by RT-PCR using random primers and the Maxima First Strand cDNA Synthesis Kit (Fermentas, St. Leon-Rot, Germany) according to the manufacturer's protocol. qPCR was then performed using specific primers to CUL5 (forward: 5'-G AACACAAGCACCCTCGTATT-3', reverse: 5'-TCAAC GGAGTTACATTCTCGTCT-3'; IDT, Leuven, Belgium) 
and actin (forward: 5'-CCAAGGCCAACCGCGAGAAGA TGAC-3', reverse: 5'-AGGGTACATGGTGGTGCCGCC AGAC-3'). CUL5 cDNA was amplified and measured using the SsoFast EvaGreen Kit (Bio-Rad, Hercules, CA, USA) according to the manufacturer's protocol. Cycling conditions were $95^{\circ} \mathrm{C}\left(30 \mathrm{~s}\right.$, activation), $95^{\circ} \mathrm{C}(5 \mathrm{~s}$, denaturation) and $60^{\circ} \mathrm{C}(10 \mathrm{~s}$, annealing/extension) for 40 cycles for CUL5 amplification on a Bio-Rad CFX96 RealTime System run on a C1000 Thermal Cycler platform (Bio-Rad, Hercules, CA, USA). Actin cDNA served as reference for relative quantification.

\section{Immunohistochemistry}

Briefly, sections from a commercially available tissue microarray (US Biomax) were deparaffinized in xylene, rehydrated in a graded ethanol series and boiled in a microwave oven for $30 \mathrm{~min}$ in citrate buffer $(\mathrm{pH}$ 6.0) followed by blocking and incubation with a primary antiCUL5 antibody (Sigma, at a 1:50 dilution). Immunodetection of the primary antibody was performed using the HistoStain PLUS kit (Invitrogen, Germany) according to the manufacturer's recommendations.

\section{Cell cycle analysis}

For cell cycle analysis, U-2 OS/centrin-GFP cells were transfected with siRNA duplexes against CUL5 mRNA (see above) and assayed for cell cycle distribution after propidium iodide staining at $72 \mathrm{~h}$ post transfection. Briefly, cells were trypsinized and pelleted by centrifugation. The cell pellet was then washed two times with PBS prepared without calcium or magnesium. After washing in PBS, the cells were resuspended in $70 \%$ ethanol and fixed overnight at $4{ }^{\circ} \mathrm{C}$. The next day, the cells were again pelleted and washed two times with $\mathrm{PBS} / 1 \%$ bovine serum albumin (BSA) to prevent clumping. After the final centrifugation, the cell pellet was suspended in $800 \mu \mathrm{PBS} / 1 \%$ BSA. The cells were then mixed with $100 \mu \mathrm{l}$ propidium iodide $(0.5 \mathrm{mg} / \mathrm{ml})$ and $100 \mu \mathrm{l}$ boiled RNase A $(10 \mathrm{mg} / \mathrm{ml})$ and incubated at $37^{\circ} \mathrm{C}$ for $30 \mathrm{~min}$. The propidium iodidestained cells were protected from light and DNA content was analyzed on a FACSCalibur flow cytometer.

\section{Comet assay}

For the analysis of DNA damage in CUL5-deficient cells, U-2 OS/centrin-GFP cells were transfected with siRNA duplexes against CUL5 mRNA (see above for protocol) for $72 \mathrm{~h}$ and efficiency of DNA repair was analyzed with or without $10 \mathrm{~Gy}$ IR using the alkaline Comet assay (Trevigen, Gaithersburg, MD, USA). Briefly, cells were trypsinized and pelleted by centrifugation. The cell pellet was then washed two times with PBS prepared without calcium or magnesium and cells were resuspended at a concentration of $1 \times 10^{5}$ cells $/ \mathrm{ml}$ in PBS. Cells were then mixed with LMAgarose at a 1:10 ratio and $50 \mu \mathrm{l}$ of this solution was pipetted onto Comet assay slides. The slides were placed at $4{ }^{\circ} \mathrm{C}$ in the dark for 10 min until the cell/agarose solution hardened. The slides were then immersed in cold lysis solution provided with the kit for $1 \mathrm{~h}$ at $4{ }^{\circ} \mathrm{C}$ in the dark. Slides were subsequently immersed in alkaline unwinding buffer $(30 \mathrm{mM} \mathrm{NaOH}, 1 \mathrm{mM}$ EDTA) for $1 \mathrm{~h}$ at $4{ }^{\circ} \mathrm{C}$ in the dark. Next, alkaline electrophoresis was performed using alkaline electrophoresis solution ( $300 \mathrm{mM} \mathrm{NaOH}, 1 \mathrm{mM}$ EDTA) at $300 \mathrm{~mA}$ for $30 \mathrm{~min}$. Slides were washed two times for $5 \mathrm{~min}$ each in $\mathrm{dH}_{2} \mathrm{O}$ and once for $5 \mathrm{~min}$ in $70 \%$ ethanol before being stained with SYBR Gold and analyzed on an Olympus AX70 epifluorescence microscope equipped with a SpotRT digital camera.

\section{TCGA data and statistical analysis}

RNA-Sequencing (RNA-Seq) and clinical data of clear cell renal cell carcinoma (KIRC) were downloaded from TCGA data portal (https://tcga-data.nci.nih.gov/tcga) on 10 December 2014. Correlations between genomic and clinical data were performed as previously described ${ }^{46}$. Briefly, the RNA-Seq Expectation-Maximization (RSEM) normalization method was used for the gene expression analysis. Gene expression was stratified based on quartiles (1st quartile $=$ low expression, 2 nd and 3rd quartiles $=$ intermediate expression, 4th quartile $=$ high expression). To compute overall survival, the patient date of death of any cause or the last date the patient was known to be alive was considered. IBM SPSS Statistics v25 was used to calculate the Kaplan-Meier survival curves, log-rank tests and univariate and multivariate Cox regression models. For all other analyses, Student's $t$-test for independent samples (two-tailed) was used wherever applicable. Three independent replicates were performed for all experiments or indicated otherwise.

\footnotetext{
Acknowledgements

We would like to thank the flow cytometry facility of the German Cancer Research Center for technical help and support. We are grateful to Rolando Cuevas for excellent technical assistance. This work was supported by the Postdoctoral Fellowship Program of the Faculty of Medicine Heidelberg (to N.K.) and the NCT-Heidelberg School of Oncology (NCT-HSO) Program (to S.P.L.).
}

\footnotetext{
Author details

${ }^{1}$ Section of Molecular Urooncology, Department of Urology, University of Heidelberg School of Medicine, Im Neuenheimer Feld 517, D-69120 Heidelberg, Germany. ${ }^{2}$ Department of Translational Medical Oncology, National Center for Tumor Diseases (NCT) Heidelberg, German Cancer Research Center (DKFZ), Im Neuenheimer Feld 460, D-69120 Heidelberg, Germany. ${ }^{3}$ Division of Applied Functional Genomics, German Cancer Research Center (DKFZ), Im Neuenheimer Feld 581, D-69120 Heidelberg, Germany. ${ }^{4}$ Department of Urology, University of Heidelberg School of Medicine, Im Neuenheimer Feld 110, D-69120 Heidelberg, Germany. ${ }^{5}$ Cancer Therapeutics Program, UPMC Hillman Cancer Center and Department of Pathology, University of Pittsburgh School of Medicine, UPMC Hillman Cancer Center, 5117 Centre Avenue, Pittsburgh, PA 15213, USA. ${ }^{6}$ Present address: Division of Solid Tumor Translational Oncology, West German Cancer Center (WTZ), Essen University Hospital, German Cancer Consortium (DKTK) at Essen/Düsseldorf, Hufelandstrasse 55, D-45147 Essen, Germany
} 


\section{Conflict of interest}

The authors declare that they have no conflict of interest.

\section{Publisher's note}

Springer Nature remains neutral with regard to jurisdictional claims in published maps and institutional affiliations.

Supplementary Information accompanies this paper at (https://doi.org/ 10.1038/541389-018-0110-2).

Received: 23 July 2018 Revised: 25 October 2018 Accepted: 10 December 2018

Published online: 09 January 2019

\section{References}

1. Shaw, G. The silent disease. Nature 537, S98-S99 (2016).

2. Frew, I. J. \& Moch, H. A clearer view of the molecular complexity of clear cell renal cell carcinoma. Annu. Rev. Pathol. 10, 263-289 (2015).

3. Turajic, S. et al. Deterministic evolutionary trajectories influence primary tumor growth: TRACERx Renal. Cell 173, 595-610e11 (2018).

4. Mitchell, T. J. et al. Timing the landmark events in the evolution of clear cell renal cell cancer: TRACERx Renal. Cell 173, 611-23e17 (2018).

5. Kovacs, G. et al. The Heidelberg classification of renal cell tumours. J. Pathol. 183, 131-133 (1997).

6. Turajic, S. et al. Tracking cancer evolution reveals constrained routes to metastases: TRACERx Renal. Cell 173, 581-94e12 (2018).

7. Cancer Genome Atlas Research Network. Comprehensive molecular characterization of clear cell renal cell carcinoma. Nature 499, $43-49$ (2013).

8. Gerlinger, M. et al. Genomic architecture and evolution of clear cell renal cell carcinomas defined by multiregion sequencing. Nat. Genet. 46, 225-233 (2014).

9. Gerlinger, M. et al. Intratumor heterogeneity and branched evolution revealed by multiregion sequencing. N. Engl. J. Med. 366, 883-892 (2012).

10. Korzeniewski, N., Spardy, N., Duensing, A. \& Duensing, S. Genomic instability and cancer: lessons learned from human papillomaviruses. Cancer Lett. 305, 113-122 (2011).

11. Thoma, C. R. et al. VHL loss causes spindle misorientation and chromosome instability. Nat. Cell Biol. 11, 994-1001 (2009).

12. Metcalf, J. L. et al. K63-ubiquitylation of VHL by SOCS1 mediates DNA doublestrand break repair. Oncogene 33, 1055-1065 (2014).

13. Kaelin, W. G. Jr. The von Hippel-Lindau tumour suppressor protein: $\mathrm{O} 2$ sensing and cancer. Nat. Rev. Cancer 8, 865-873 (2008).

14. Cardote, T. A. F., Gadd, M. S. \& Ciulli, A. Crystal structure of the Cul2-Rbx1-EloBCVHL ubiquitin ligase complex. Structure 25, 901-11e3 (2017).

15. Petroski, M. D. \& Deshaies, R. J. Function and regulation of cullin-RING ubiquitin ligases. Nat. Rev. Mol. Cell Biol. 6, 9-20 (2005).

16. Okumura, F., Joo-Okumura, A., Nakatsukasa, K. \& Kamura, T. The role of cullin 5containing ubiquitin ligases. Cell Div. 11, 1 (2016).

17. Sarikas, A., Hartmann, T. \& Pan, Z. Q. The cullin protein family. Genome Biol. 12, 220 (2011).

18. Pena-Llopis, S. et al. BAP1 loss defines a new class of renal cell carcinoma. Nat. Genet. 44, 751-759 (2012).

19. $\mathrm{Yu}, \mathrm{H}$. et al. Tumor suppressor and deubiquitinase BAP1 promotes DNA double-strand break repair. Proc. Natl. Acad. Sci. USA 111, 285-290 (2014).

20. Ge, Z. et al. Integrated genomic analysis of the ubiquitin pathway across cancer types. Cell Rep. 23, 213-26e3 (2018).

21. Duensing, A. et al. Centriole overduplication through the concurrent formation of multiple daughter centrioles at single maternal templates. Oncogene 26, 6280-6288 (2007).

22. Habedanck, R., Stierhof, Y. D., Wilkinson, C. J. \& Nigg, E. A. The Polo kinase Plk4 functions in centriole duplication. Nat. Cell Biol. 7, 1140-1146 (2005).
23. Jin, J., Ang, X. L., Shirogane, T. \& Wade Harper, J. Identification of substrates for F-box proteins. Methods Enzymol. 399, 287-309 (2005).

24. Guarguaglini, G. et al. The forkhead-associated domain protein Cep170 interacts with Polo-like kinase 1 and serves as a marker for mature centrioles. Mol. Biol. Cell 16, 1095-1107 (2005).

25. Petroski, M. D. \& Deshaies, R. J. Function and regulation of cullin-RING ubiquitin ligases. Nat. Rev. Mol. Cell Biol. 6, 9-20 (2005).

26. Mahrour, N. et al. Characterization of Cullin-box sequences that direct recruitment of Cul2-Rbx1 and Cul5-Rbx2 modules to Elongin BC-based ubiquitin ligases. J. Biol. Chem. 283, 8005-8013 (2008).

27. Brodaczewska, K. K., Szczylik, C., Fiedorowicz, M., Porta, C. \& Czarnecka, A. M. Choosing the right cell line for renal cell cancer research. Mol. Cancer 15, 83 (2016).

28. Lewis, S. P., Willis, A. N., Johnson, A. E., Resau, J. \& Burnatowska-Hledin, M. A. Mutational analysis of VACM-1/cul5 exons in cancer cell lines. APMIS $\mathbf{1 1 9}$ 421-430 (2011).

29. Burnatowska-Hledin, M. A. et al. Expression cloning of an AVP-activated, calcium-mobilizing receptor from rabbit kidney medulla. Am. J. Physiol. 268(6 Pt 2), F1198-F1210 (1995).

30. Byrd, P. J. et al. Identification and analysis of expression of human VACM-1, a cullin gene family member located on chromosome 11q22-23. Genome Res. 7, $71-75$ (1997).

31. Mathias, N. et al. Cdc53p acts in concert with $C d c 4 p$ and $C d c 34 p$ to control the G1-to-S-phase transition and identifies a conserved family of proteins. Mol. Cell. Biol. 16, 6634-6643 (1996).

32. Kipreos, E. T., Lander, L. E., Wing, J. P., He, W. W. \& Hedgecock, E. M. cul-1 is required for cell cycle exit in $\mathrm{C}$. elegans and identifies a novel gene family. Cell 85, 829-839 (1996).

33. $\mathrm{Yu}, \mathrm{X}$. et al. Induction of APOBEC3G ubiquitination and degradation by an HIV1 Vif-Cul5-SCF complex. Science 302, 1056-1060 (2003).

34. Fay, M. J. et al. Analysis of CUL-5 expression in breast epithelial cells, breast cancer cell lines, normal tissues and tumor tissues. Mol. Cancer 2, 40 (2003).

35. Kalla, C. et al. Analysis of 11q22-q23 deletion target genes in B-cell chronic lymphocytic leukaemia: evidence for a pathogenic role of NPAT, CUL5, and PPP2R1B. Eur. J. Cancer 43, 1328-1335 (2007).

36. Devor, E. J. et al. Cullin-5, a ubiquitin ligase scaffold protein, is significantly underexpressed in endometrial adenocarcinomas and is a target of miR-182. Oncol. Rep. 35, 2461-2465 (2016).

37. Xu, X. M. et al. MicroRNA-19a and $-19 \mathrm{~b}$ regulate cervical carcinoma cell proliferation and invasion by targeting CUL5. Cancer Lett. 322, 148-158 (2012).

38. Kunkler, B. et al. CUL5 is required for thalidomide-dependent inhibition of cellular proliferation. PLoS One 13, e0196760 (2018).

39. Scelo, G. et al. Genome-wide association study identifies multiple risk loci for renal cell carcinoma. Nat. Commun. 8, 15724 (2017).

40. Burrell, R. A. et al. Replication stress links structural and numerical cancer chromosomal instability. Nature 494, 492-496 (2013).

41. Buchwalter, A. et al. Expression of VACM-1/cul5 mutant in endothelial cells induces MAPK phosphorylation and maspin degradation and converts cells to the angiogenic phenotype. Microvasc. Res. 75, 155-168 (2008).

42. Laszlo, G. S. \& Cooper, J. A. Restriction of Src activity by Cullin-5. Curr. Biol. 19 157-162 (2009).

43. Roche, S., Fumagalli, S. \& Courtneidge, S. A. Requirement for Src family protein tyrosine kinases in G2 for fibroblast cell division. Science 269, 1567-1569 (1995).

44. Fukumoto, Y. et al. Src family kinases promote silencing of ATR-Chk1 signaling in termination of DNA damage checkpoint. J. Biol. Chem. 289, 12313-12329 (2014).

45. Piel, M., Meyer, P., Khodjakov, A., Rieder, C. L. \& Bornens, M. The respective contributions of the mother and daughter centrioles to centrosome activity and behavior in vertebrate cells. J. Cell. Biol. 149, 317-330 (2000).

46. Pena-Llopis, S., Wan, Y. \& Martinez, E. D. Unique epigenetic gene profiles define human breast cancers with poor prognosis. Oncotarget 7, 85819-85831 (2016). 